\title{
Imaging technology developments at Leti, and focus on cooled and uncooled infrared detectors
}

by Dr. François Simoens* and Dr. Olivier Gravrand ${ }^{\circ}$

* CEA-Leti, Optics \& Photonics Department, Imaging Program Manager, Grenoble, France ${ }^{\circ}$ CEA-Leti, Optics \& Photonics Department, Senior Physicist, Infrared Detection, Grenoble, France

Being a key player in imaging, CEA-Leti has built a world-class scientific, technological and human environment around its imaging platform, providing manufacturers, universities and laboratories. Leti's imaging expertise covers the infrared and the visible spectrum, and extends to the fields of millimetric, terahertz and X-ray imaging. Active in rupture-imaging concepts ranging from growth of the base materials to characterization of the final imagers, and including the integration of optics and on chip functions and processing, Leti's imaging platform offers a unique range of imaging opportunities.

This presentation will begin by a wide introduction of the Leti R\&D work in imaging. Then a particular emphasis will be put on the latest achievements in cooled and uncooled infrared focal plane array developments. Finally, latest issues will be discussed with regards to applications like thermography and non-destructive testing. 